\title{
Tumour angiogenesis and repulsive guidance molecule b: $A$ role in HGF- and BMP-7-mediated angiogenesis
}

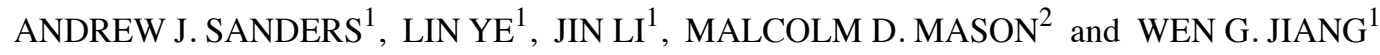 \\ ${ }^{1}$ Cardiff University-Peking University Cancer Institute and ${ }^{2}$ Section of Oncology \\ and Palliative Medicine, Cardiff University School of Medicine, Cardiff, UK
}

Received April 7, 2014; Accepted May 26, 2014

DOI: $10.3892 /$ ijo.2014.2508

\begin{abstract}
Hepatocyte growth factor (HGF) is a key growth factor linked to promoting cancer progression and angiogenesis. The present study identifies repulsive guidance molecule $b$ (RGMb), a bone morphogenetic protein (BMP) co-receptor, as a gene whose expression is regulated by HGF and explores the potential of RGMb to contribute to the process of angiogenesis. Microarray analysis was used to identify HGF responsive genes in HECV endothelial cells, identifying RGMb. RGMb was subsequently targeted using a ribozyme transgene system and its role in angiogenesis assessed using in vitro and in vivo assays. The importance of RGMb in pro-angiogenic responses to HGF and BMP-7 was also assessed. Microarray analysis identified RGMb as a gene upregulated as a result of HGF treatment. Knockdown of RGMb, in HECV cells, had minimal effects on tubule formation, brought about a general, although non-significant increase in cell growth and enhanced cell migration. Similarly, no significant effect of RGMb knockdown was found in vivo using a co-inoculation angiogenesis model. Knockdown of RGMb was, however, found to reduce the responsiveness of $\mathrm{HECV}$ cells to HGF treatment and particularly to BMP-7 treatment in regard to the enhanced migratory and tubule formation brought about by these treatments in vitro. Our results indicate that RGMb expression can be influenced by HGF treatment. Whilst this molecule appears to have minimal impact on angiogenic traits individually, it demonstrates an involvement in propagating pro-angiogenic effects of HGF and particularly BMP-7 and thus, may play a role in regulating angiogenic responses to HGF and BMP-7.
\end{abstract}

\section{Introduction}

The field of angiogenesis and its potential as a target to slow cancer development is well established and reviewed within

Correspondence to: Dr Andrew J. Sanders, Cardiff UniversityPeking University Cancer Institute, Cardiff University School of Medicine, Cardiff, CF14 4XN, UK

E-mail: sandersaj1@cf.ac.uk

Key words: RGMb, HGF, BMP-7, tubule formation, tumour angiogenesis the literature. Angiogenesis is an essential process required for normal physiological events such as wound healing, reproduction, development and immunity. However, imbalance of this process is often seen in disease states such as cancer progression and metastatic spread, where enhanced tumour vasculature facilitates rapid tumour growth and provides access for metastasising cells $(1,2)$. Numerous pro-angiogenic factors have been identified. One such molecule is hepatocyte growth factor (HGF), which has long been established as a factor that can enhance the aggressive nature of cancer cells through its ability to promote pro-metastatic traits such as motogenesis, mitogenesis, morphogenesis and angiogenesis (3). HGF is able to promote angiogenesis directly through its motogenic and morphogenic impact on endothelial cells and also indirectly, through its ability to enhance the production of other pro-angiogenic factors such as vascular endothelial grow th factor (VEGF) $(3,4)$. Previous studies have demonstrated the anti-angiogenic potential of targeting HGF in breast and prostate cancer model systems $(5,6)$. The present study aimed to identify gene expression in endothelial cells that could be regulated by HGF which may subsequently contribute to the process of HGF-promoted angiogenesis, identifying repulsive guidance molecule b (RGMb) as one such molecule.

Following the establishment of RGM as a gene involved in the guidance of chick retinal axons (7), three mouse orthologues (termed RGMa, RGMb and RGMc) were isolated and characterised in separate studies, displaying predominant expression in the developing and adult nervous system (RGMa and RGMb) and skeletal muscles (RGMc) (8-10). An independent study, conducted at the same time, also identified DRAGON (RGMb) using a genome binding strategy to screen for DRG11 regulated genes (11).

RGMb shares structural similarities with the other members of the RGM family, including a N-terminal signal peptide, a proteolytic cleavage site, a von Willebrand factor (vWF) type D domain and a glycophosphatidylinositol (GPI) anchor, and is expressed in the developing and adult nervous system (7-11). RGMb has also been shown to be involved in promoting mouse dorsal root ganglion (DRG) neuron adhesion (11), has been implicated in axonal regeneration after injury (12) and in response to spinal cord injury, where it is found to be upregulated (13). In addition to its roles in the nervous system, RGMb has also been shown to contribute 
to bone morphogenetic protein (BMP) signalling, where it has been identified, together with RGMa and RGMc, as a BMP co-receptor (14-17). Additionally, RGMb may play an important role in reproduction through the enhancement of BMP signalling (18) and a potential role for RGMb has also been demonstrated in the immune system where it can inhibit IL-6 expression in macrophages through the p38 MAPK and ERK1/2 pathway, but not the Smad $1 / 5 / 8$ pathway, in a BMP-ligand-dependent manner (19).

A number of recent studies from our laboratories have explored the importance of the members of the RGM family in breast and prostate cancer (20-22). RGM levels were examined in a breast cancer cohort in conjunction with clinical pathological patient data. This study highlighted an aberrant expression profile of the RGMs in breast cancer, indicating potential links between RGMa and RGMb and patient prognosis (20). Further work explored the impact of targeting RGMb in breast cancer cell lines in vitro. This study demonstrated a potential role for RGMb in breast cancer proliferation, matrix-adhesion and migration where targeting this molecule enhanced these aggressive traits and implicated links with Smad-dependent and Smad-independent pathways (22). Similarly, RGMb appears to play a role in prostate cancer progression. Knockdown of RGMb in the PC-3 prostate cancer cell line enhanced cell growth and migratory rates and also increased cell-matrix adhesion. Additionally, RGMb may be linked to Smad signalling in this cell line as knockdown of RGMb appeared to affect the levels of activated Smad 1 and 3, and also enhanced ID1 expression (21). Taken together these studies suggest important roles for RGMb in breast and prostate cancer progression and also imply an involvement in BMP and Smad signalling.

In the present study, the potential involvement of $\mathrm{RGMb}$ was explored in the process of tumour angiogenesis through the targeting of RGMb in HECV endothelial cells. Suppression of RGMb in HECV cells did not result in significant changes to a number of traits associated with the angiogenic process. However, we report that suppression of RGMb in HECV cells can act to decrease or inhibit the pro-angiogenic effect brought about by HGF and, more substantially, BMP-7 on tubule formation and migration rates. This study highlights the potential importance of RGMb in propagating pro-angiogenic effects of HGF and BMP-7.

\section{Materials and methods}

Reagents, cell lines and culture conditions. Human HECV endothelial cells were purchased from Interlab Cell Line Collection (ICLC, Naples, Italy). The MCF-7 breast cancer cell line and the PC-3 human prostate cancer cell line were purchased from the American Type Culture Collection (ATCC, Rockville, MD, USA). Hepatocyte growth factor (HGF) was a kind gift from Dr T. Nakamura (Osaka University Medical School, Osaka, Japan) and bone morphogenetic protein-7 (BMP-7) was purchased from Sigma (Dorset, UK). All cells were maintained in Dubecco's modified Eagle's medium (DMEM) (PAA Laboratories Ltd., Somerset, UK), supplemented with penicillin, streptomycin and $10 \%$ fetal calf serum (PAA Laboratories Ltd.) and incubated at $37^{\circ} \mathrm{C}, 5 \% \mathrm{CO}_{2}$ and $95 \%$ humidity.
Generation of human HECV endothelial cells displaying suppressed RGMb expression. RGMb expression was targeted in human HECV endothelial cells using a ribozyme transgene specifically generated to target and cleave RGMb transcript. This methodology has been previously reported $(23,24)$. Plasmids containing ribozyme transgenes had previously been developed within the laboratory $(21,22)$. Briefly, ribozyme transgene sequences were designed based on the predicted secondary structure of the RGMb transcript using Zukers RNA Mfold program (25) and were synthesised by Invitrogen (Paisley, UK). Ribozyme transgenes were subsequently cloned into a pEF6/V5-His-TOPO plasmid vector (Invitrogen). Both control pEF6 plasmids containing no insert, and plasmids containing the RGMb ribozyme transgene were transfected into HECV cells using electroporation. Following transfection, these cells underwent a selection period and subsequent verification of RGMb knockdown. Cells containing the RGMb ribozyme transgene were termed HECV ${ }^{\text {RGMb KO }}$ and were compared throughout the study to control HECV cells containing closed plasmids alone, termed HECV ${ }^{\mathrm{pEF}}$.

RNA extraction and reverse transcription-polymerase chain reaction $(R T-P C R)$. Cells were grown to confluence in a $25-\mathrm{cm}^{2}$ flask before RNA was extracted using total RNA isolation (TRI) reagent (Sigma) in accordance with the supplied protocol. RNA was subsequently quantified using a spectrophotometer (WPA UV 1101, Biotech Photometer, Cambridge, UK), standardised to a concentration of $500 \mathrm{ng}$ and used as a template to generate cDNA using an iScript cDNA synthesis kit (Bio-Rad Laboratories Ltd., Hemel Hempstead, UK). Following cDNA synthesis, sample quality and uniformity was checked, using $\beta$-actin primers, before assessing RGMb expression using specific primers for RGMb transcript (full primer details are shown in Table I). PCR was performed using REDTaq ${ }^{\circledR}$ ReadyMix $^{\mathrm{TM}}$ PCR Reaction Mix (Sigma). The following reaction conditions were set up in a T-Cy Thermocycler (Creacon Technologies Ltd., The Netherlands); denaturing at $94^{\circ} \mathrm{C}$ for $40 \mathrm{sec}$, annealing at $55^{\circ} \mathrm{C}$ for $40 \mathrm{sec}$ and extension at $72^{\circ} \mathrm{C}$ for $60 \mathrm{sec}$. PCR was conducted over 34 cycles following an initial 5-min denaturing step $\left(94^{\circ} \mathrm{C}\right)$ and concluded with a final 10 -min extension step $\left(72^{\circ} \mathrm{C}\right)$. Amplified products were loaded onto an agarose gel, separated electrophoretically, stained in ethidium bromide and visualised under ultraviolet light.

Microarray analysis of gene expression. Two sets of triplicate HECV cell flasks were treated with either $40 \mathrm{ng} / \mathrm{ml}$ hepatocyte growth factor (HGF) for $4 \mathrm{~h}$ or remained untreated and RNA extracted as described above using TRI reagent (Sigma). Extracted RNA was quantified before being sent to the Cardiff University Central Biotechnology Services (CBS) microarray facility for labelling and hybridisation to a GeneChip Human Genome U133 Plus 2.0 array (Affymetrix UK Ltd., High Wycombe, UK).

Quantitative polymerase chain reaction ( $q P C R$ ). Quantitative PCR was used to assess RGMb transcript levels in control and transfected cell lines following a previously reported method $(26,27)$. Briefly, the iCycler IQ system was used to detect and quantify RGMb transcript expression in each sample. 
Table I. Primers used in the study.

\begin{tabular}{lll}
\hline Primer set & \multicolumn{1}{c}{ Sense } & \multicolumn{1}{c}{ Anti-sense } \\
\hline$\beta$-actin probe & ATGATATCGCCGCGCTCG & CGCTCGGTGAGGATCTTCA \\
RGMb probe & GGATCCAGTGCTACTGCTAC & GTAAAGTTGGCATCACCAGT \\
GAPDH qPCR & CTGAGTACGTCGTGGAGTC & ACTGAACCTGACCGTACACAGAGATGATGACCCTTTTG \\
RGMb qPCR & TTCAGTTCAAGTGACAAACG & ACTGAACCTGACCGTACATCATCTGTCACAGCTTGGTA \\
\hline
\end{tabular}

ACTGAACCTGACCGTACA indicates $\mathrm{Z}$ sequence.

Transcript copy number was calculated based on an internal standard. Samples were normalised against GAPDH expression (see Table I for primer details). The Amplifluor system (Intergen Inc., New York, NY, USA) was utilised together with qPCR Master Mix (ABgene, Surrey, UK). Conditions for qPCR were; $15-\mathrm{min}$ initial $95^{\circ} \mathrm{C}$ period followed by 60 cycles of $95^{\circ} \mathrm{C}$ for $15 \mathrm{sec}, 55^{\circ} \mathrm{C}$ for $60 \mathrm{sec}$ and $72^{\circ} \mathrm{C}$ for $20 \mathrm{sec}$.

SDS-PAGE and western blotting. Protein was extracted from a confluent $75-\mathrm{cm}^{2}$ tissue culture flask. Cells were detached and lysed in HCMF buffer containing $0.5 \%$ SDS, $1 \%$ Triton X-100, $2 \mathrm{mM} \mathrm{CaCl}_{2}, 100 \mu \mathrm{g} / \mathrm{ml}$ phenylmethylsulfonyl fluoride, $1 \mathrm{mg} / \mathrm{ml}$ leupeptin, $1 \mathrm{mg} / \mathrm{ml}$ aprotinin and $10 \mathrm{mM}$ sodium orthovanadate on a rotor wheel for $1 \mathrm{~h}$ before removal of insolubles through centrifugation at 13,000 g. The Bio-Rad DC Protein assay kit (Bio-Rad Laboratories, CA, USA) was used to quantify protein levels in the samples. Samples were subsequently standardised to $2 \mathrm{mg} / \mathrm{ml}$ and diluted in Laemmli $2 \mathrm{X}$ concentrate sample buffer (Sigma) before being boiled for $5 \mathrm{~min}$. Samples were loaded onto a $10 \%$ acrylamide gel and separated electrophoretically. Following separation the proteins were blotted onto a Hybond-C Extra nitrocellulose membrane (Amersham Biosciences UK Ltd., Bucks, UK) and blocked in $10 \%$ milk. RGMb expression was detected using anti-RGMb antibody (Santa Cruz Biotechnology, Inc., Santa Cruz, CA, USA). In addition to this, GAPDH expression was also detected, to assess sample uniformity, using an anti-GAPDH antibody (Santa Cruz Biotechnology, Inc.). Following binding of the primary antibody, the membranes were probed with peroxidase conjugated anti-rabbit (RGMb) or anti-mouse (GAPDH) secondary antibodies (Sigma). Expression was then visualised through the Supersignal West Dura Extended Duration substrate chemi-luminescent system (Perbio Science UK Ltd., Cramlington, UK) and detected using a UVIProChem camera system (UVItec Ltd., Cambridge, UK).

In vitro cell growth assay. An in vitro growth assay was used to examine the impact of RGMb on cell growth. Cells were seeded into 96-well plates at a density of 3,000 cells/well and triplicate plates were set up to allow overnight, 3- and 5-day incubation periods. Following incubation, the plates were fixed in $4 \%$ formaldehyde (v/v) and stained with $0.5 \%(\mathrm{w} / \mathrm{v})$ crystal violet. Subsequently, $10 \%$ acetic acid (v/v) was used to extract the crystal violet stain and cell density was detected through spectrophotmeric analysis using a Bio-Tek ELx800 multi-plate reader (Bio-Tek Instruments Inc., VT, USA).
In vitro cell migration/wounding assay. Cellular migration was assessed using a migration/wounding assay modified from a previously described method (28). Briefly, cells were cultured to confluence in a 24-well plate before scratching the cell monolayer with a pointed plastic pipette tip. Subsequently, wound closure, through the migration of cells, was tracked and photographed at 15 -min interval time-points over a 90-min period using an inverted microscope and GXcapture software. The distance between the two wound fronts was calculated at several consistent points over all the time intervals using Image J software and average cellular migration calculated.

In vitro tubule formation assay. The potential of RGMb to contribute to the process of HECV tubule formation was assessed in vitro using a Matrigel endothelial cell tubule formation assay modified from a previously reported study (29). Briefly, $500 \mu \mathrm{g}$ of Matrigel, diluted in serum-free medium, was seeded into a 96-well plate and incubated for a minimum of $40 \mathrm{~min}$ to allow setting of the Matrigel. Once set, $35,000 \mathrm{HECV}$ endothelial cells (HECV ${ }^{\mathrm{pEF} 6}$ or HECV ${ }^{\mathrm{RGMb} \text { KO }}$ cells) were seeded onto the Matrigel layer and incubated for 4-5 h. Tubule formation occurring over the incubation period was visualised under low magnification and images captured for analysis. Total tubule perimeter per field was subsequently quantified using ImageJ software. Where appropriate, treatments consisting of either $40 \mathrm{ng} / \mathrm{ml} \mathrm{BMP-7}$ or $40 \mathrm{ng} / \mathrm{ml} \mathrm{HGF}$ were added to the cell medium following seeding.

In vivo tumour development assay. The impact of RGMb suppression in vivo was examined using a previously described in vivo angiogenesis model (29). Briefly, a 100- $\mu$ l suspension containing $1 \times 10^{6}$ cancer cells (either MCF-7 ${ }^{\mathrm{pEF} 6}$ breast cancer cells or $\mathrm{PC}-3^{\mathrm{pEF} 6}$ prostate cancer cells) were subcutaneously injected into the left and right flanks of 4-6-week old athymic nude mice (CD-1; Charles River Laboratories, Kent, UK) together with either $1 \times 10^{6} \mathrm{HECV}^{\mathrm{pEF} 6}$ or $1 \times 10^{6} \mathrm{HECV}^{\mathrm{RGMb} \mathrm{KO}}$ cells in a $0.5 \mathrm{mg} / \mathrm{ml}$ Matrigel solution. The tumours were allowed to develop over the course of the experiment and were measured twice weekly using vernier callipers under sterile conditions. The mice were housed in filter top units and were treated humanely in accordance with United Kingdom Home Office and the United Kingdom Coordinating Committee on Cancer Research (UKCCCR) guidelines. All in vivo work undertaken in this study was conducted under the project license (PPL 30/2591) of the British Home Office. Animals were dispatched humanely if severity limits were reached or at the experimental end point using a schedule 1 method. Tumour 


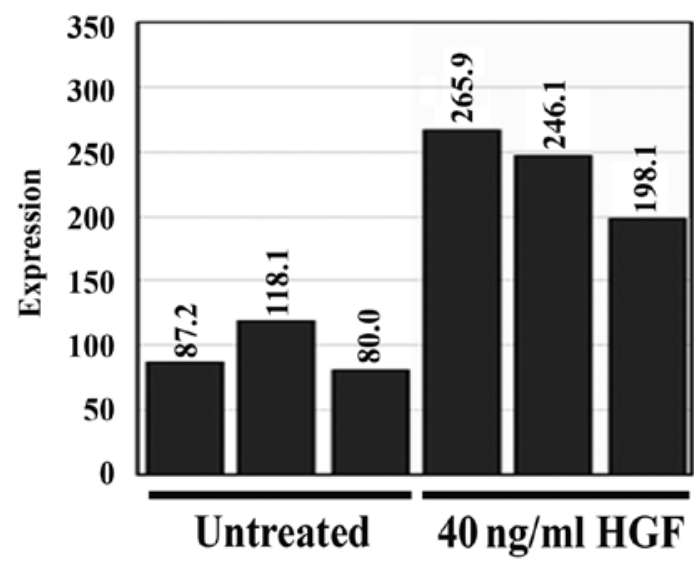

Figure 1. Treatment of HECV endothelial cells with HGF upregulates RGMb expression. Microarray analysis demonstrated a significant increase in RGMb expression in triplicate samples treated with $40 \mathrm{ng} / \mathrm{ml} \mathrm{HGF}$ in comparison to untreated samples $(\mathrm{p}=0.004)$

volume was calculated for each time-point using the following formula: Tumour volume $=0.523 \mathrm{x}$ width $^{2} \mathrm{x}$ length.

Statistical analysis. The Minitab 14 statistical package was used to test for statistical differences between RGMb knockdown HECV cells and the pEF6 vector control HECV cells using a two sample, two tailed t-test. Experimental procedures were repeated a minimum of three independent times. Data represents mean values \pm SEM, values of $p<0.05$ were regarded as statistically significant.

\section{Results}

Hepatocyte growth factor can regulate repulsive guidance molecule $b$ expression. A microarray study was conducted to examine how gene expression within the HECV human endothelial cell line was affected following 4-h treatment with $40 \mathrm{ng} / \mathrm{ml}$ HGF. Treatment with HGF caused a range of differential gene expression within this endothelial cell line. Repulsive guidance molecule b (RGMb) expression was significantly increased following HGF treatment $(\mathrm{p}=0.004$ vs. untreated control cells) and the expression of RGMb was found to be enhanced $\sim 2.5$ times by HGF treatment (Fig. 1).

Suppression of RGMb expression using a ribozyme transgene system. RGMb expression was successfully knocked down following transfection of HECV cells with a pEF6 plasmid containing a ribozyme transgene specifically targeted to $\mathrm{RGMb}$ transcript. Reduced RGMb transcript expression can be seen in the HECV ${ }^{\text {RGMb KO }}$ cells in comparison to empty plasmid control $\mathrm{HECV}^{\mathrm{pEF} 6}$ cells using RT-PCR and quantitative PCR (Fig. 2A and B). Similarly, reduced RGMb protein levels were observed, using western blot analysis, in HECV cells transfected with the ribozyme transgene in comparison to control cells (Fig. 2C).

Impact of RGMb suppression on endothelial in vitro cell traits. Following successful targeting of RGMb, the impact of this knockdown on endothelial cell functions was examined
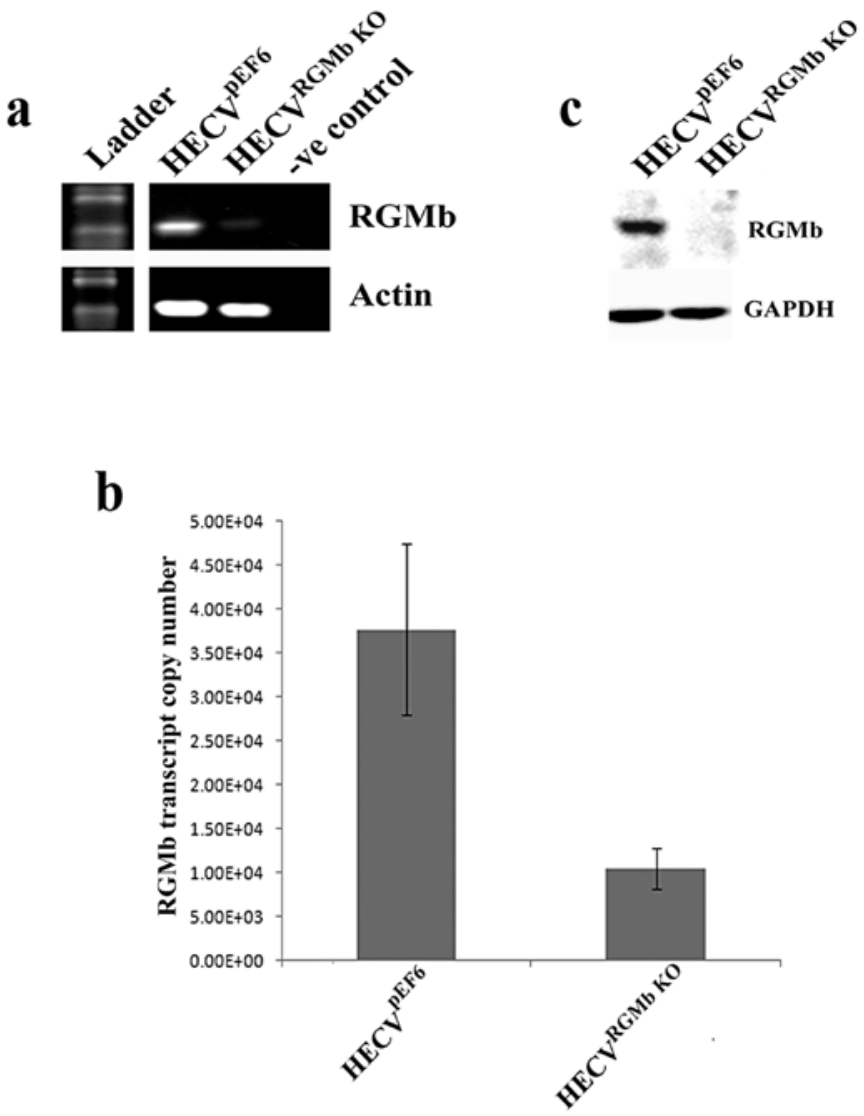

Figure 2. Verification of RGMb suppression in HECV cells. RGMb was successfully targeted and knocked down in HECV cells through transfection with the RGMb specific ribozyme transgene. Knockdown in HECV ${ }^{\text {RGM KO }}$ cells was confirmed, in comparison to the plasmid control HECV ${ }^{\mathrm{pEF} 6}$ cells, at both the transcript level, using RT-PCR (a) and qPCR (b), and the protein level, using western blot analysis (c). Error bars represent SEM.

in vitro. Knockdown of RGMb did not significantly alter the rate of cell growth in HECV cells (Fig. 3A), however, the suppression did tend to increase growth rates over both a 3 - and 5-day incubation period (HECV ${ }^{\mathrm{pEF}}$ vs. $\mathrm{HECV}^{\mathrm{RGMb} \mathrm{KO}}, \mathrm{p}=0.08$, 3 -day incubation; 5-day incubation $\mathrm{p}=0.15$ ). Suppression of RGMb appeared to impact later stages of HECV migration (Fig. 3B), with enhanced levels of migration being observed in HECV ${ }^{\mathrm{RGMb}} \mathrm{KO}$ cells compared to HECV ${ }^{\mathrm{pEF}}$ control cells at $75 \mathrm{~min}(\mathrm{p}=0.069)$ and $90 \mathrm{~min}(\mathrm{p}=0.035)$. Our results also suggest that knockdown of RGMb has little effect on HECV tubule formation and thus angiogenic potential in vitro (Fig. 3C and D), where using this angiogenic assay little difference in levels of tubule formation was observed between control $\mathrm{HECV}^{\mathrm{pEF}}$ and HECV ${ }^{\mathrm{RGMb}} \mathrm{KO}$ cells. No significant difference between quantified total tubule perimeter levels was seen between $\mathrm{HECV}^{\mathrm{p} E F 6}$ and HECV ${ }^{\mathrm{RGMb}}{ }^{\mathrm{KO}}$ cells ( $\mathrm{p}=0.813$ ).

Suppression of $R G M b$ has limited impact in vivo. We next investigated the role of RGMb knockdown using an in vivo angiogenesis model, whereby endothelial cells, either control or RGMb suppressed, were inoculated alongside cancer cells to examine their potential to impact on the development of the cancer cell tumour. This was tested in a prostate cancer model (Fig. 4A), where PC-3 cells were used and a breast 
$\mathbf{a}$

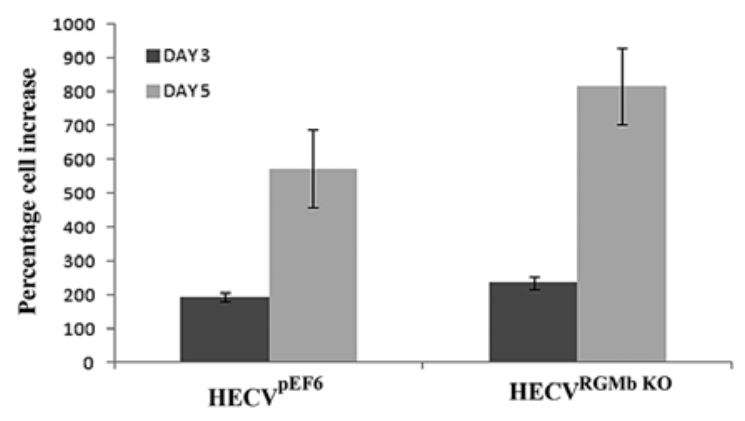

b

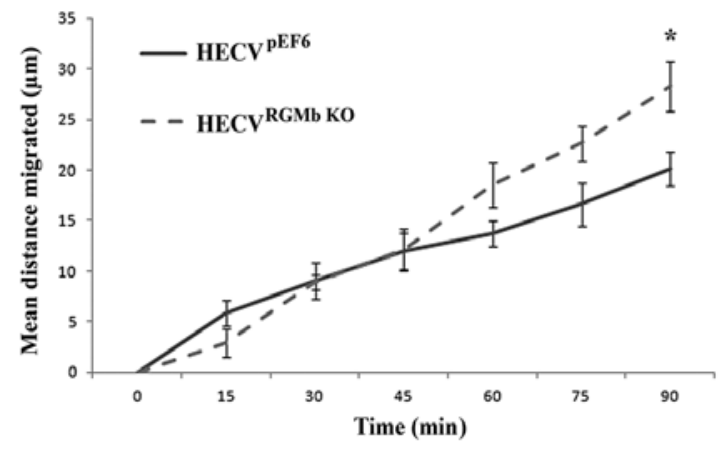

c

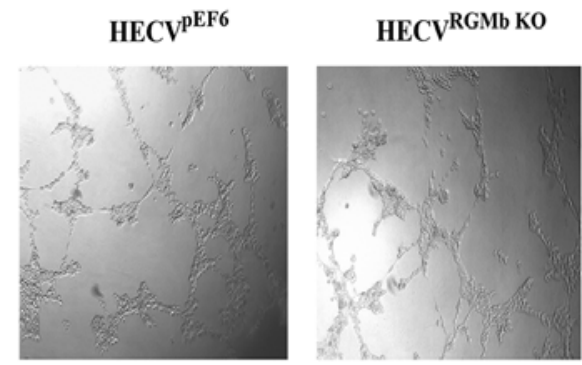

d

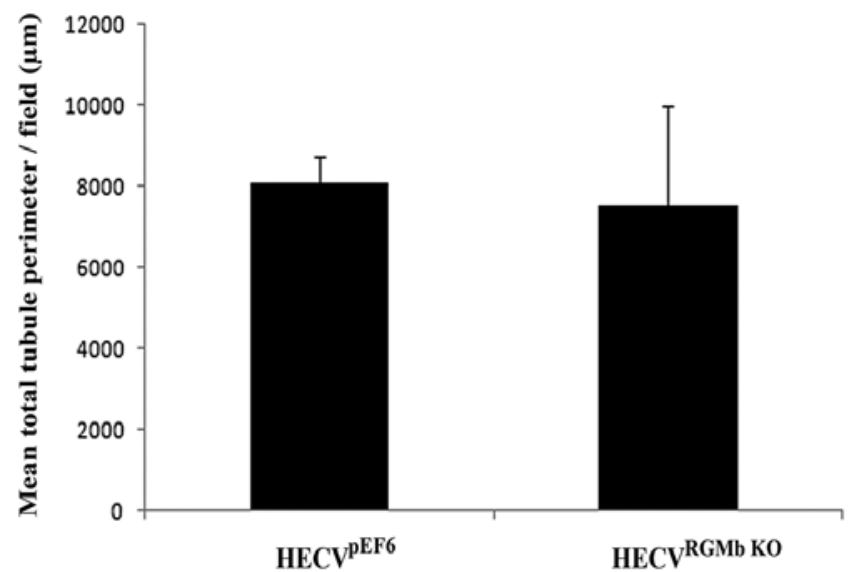

Figure 3. In vitro impact of RGMb on HECV cell function. Knockdown of RGMb tended to enhance cell growth in comparison to the HECVEF6 control cells over 3- and 5-day incubation periods, though this did not reach significant levels (a). Knockdown of RGMb did significantly enhance migrational rates in comparison to $\mathrm{HECV}^{\mathrm{pEF} 6}$ cells following $90 \mathrm{~min}$ (b), but was not seen to have any impact on angiogenic potential in a Matrigel tubule formation assay (c and d). Representative images are shown in (c). Error bars represent SEM; ${ }^{\mathrm{p}} \mathrm{p}<0.05$.

cancer model (Fig. 4B), where MCF-7 cells were used. In both cases little difference was observed between tumour development involving control HECV ${ }^{\mathrm{pEF} 6}$ or $\mathrm{HECV}^{\mathrm{RGMb} \mathrm{KO}}$ cells, with neither model yielding statistically different levels of tumour development ( $\mathrm{p}>0.05$ in both cases).

$R G M b$ suppression can limit pro-angiogenic effects of $H G F$. Following on from establishing the involvement of RGMb in traits associated with the angiogenic cascade, we next looked to identify its importance in how HGF could enhance these angiogenic traits (Fig. 5). Following treatment with $40 \mathrm{ng} / \mathrm{ml} \mathrm{HGF}$, a significant increase was seen in $\mathrm{HECV}^{\mathrm{pEF} 6}$ cell migration rates (Fig. 5A). Treatment with HGF significantly enhanced the distance migrated by $\mathrm{HECV}^{\mathrm{pEF} 6}$ cells following $60-(\mathrm{p}=0.02), 75-(\mathrm{p}=0.02)$ and $90-\mathrm{min}(\mathrm{p}=0.001)$ time-points. In contrast, when RGMb was targeted in this endothelial cell line, treatment with $40 \mathrm{ng} / \mathrm{ml} \mathrm{HGF}$ did not appear to have as great a pro-migratory effect (Fig. 5B). General increases in migration rates were observed in the later time-points of the experiment but these were not found to be statistically significant $(\mathrm{p}=0.44$ at $60 \mathrm{~min}, \mathrm{p}=0.24$ at $75 \mathrm{~min}$ and $\mathrm{p}=0.23$ at $90 \mathrm{~min}$ ). Similar trends were observed with tubule formation capacity (Fig. 5C and D). In keeping with its established pro-angiogenic role, HGF significantly enhanced the level of tubule formation in this in vitro angiogenic assay in the control $\mathrm{HECV}^{\mathrm{pEF} 6}$ cell line (untreated HECV ${ }^{\mathrm{pEF} 6}$ vs. $40 \mathrm{ng} / \mathrm{ml} \mathrm{HGF}$ treated HECV ${ }^{\mathrm{pEF}}$, $\mathrm{p}=0.001$ ). However, when $\mathrm{HECV}^{\mathrm{RGMb} \text { KO }}$ cells were treated with HGF the increase in tubule formation was not as great and no significant difference was observed between untreated and $40 \mathrm{ng} / \mathrm{ml}$ HGF treated HECV ${ }^{\text {RGMb KO }}$ cells $(\mathrm{p}=0.210)$.

RGMb suppression inhibits BMP-7 pro-angiogenic response. As the RGM family have been identified as BMP co-receptors, we also examined the impact of suppressing RGMb expression on HECV BMP promoted responses, in particular BMP-7. In control HECV ${ }^{\mathrm{pEF} 6}$ cells, treatment with BMP-7 caused a notable increase in cell migration (Fig. 6A), which was apparent in the later stages of the experiment (untreated HECV ${ }^{\mathrm{pEF} 6}$ vs. $40 \mathrm{ng} / \mathrm{ml} \mathrm{BMP-7}$ treated HECV ${ }^{\mathrm{pEF} 6} ; 60 \mathrm{~min}, \mathrm{p}=0.037 ; 75 \mathrm{~min}$, $\mathrm{p}=0.144 ; 90 \mathrm{~min}, \mathrm{p}=0.089)$. In contrast to this, suppression of RGMb in HECV endothelial cells removed the migratory response of this cell line to BMP-7 with very little difference being observed between the migration pattern of $\mathrm{HECV}^{\mathrm{RGMb} \mathrm{KO}}$ cells treated with $40 \mathrm{ng} / \mathrm{ml}$ BMP-7 and control, untreated $\mathrm{HECV}^{\text {RGMb KO }}$ cells (Fig. 6B). A similar trend was also apparent in the tubule formation experiments (Fig. 6C and D). In control $\mathrm{HECV}^{\mathrm{pEF} 6}$ cells, treatment with $40 \mathrm{ng} / \mathrm{ml}$ BMP-7 caused an increase in the tubule formation potential of these endothelial cells and treatment of HECV ${ }^{\mathrm{pEF} 6}$ cells with $40 \mathrm{ng} / \mathrm{ml}$ BMP-7 brought about a significant increase in total tubule perimeter $(\mathrm{p}=0.005)$. As with cell migration, suppression of RGMb in HECV cells removed the cells responsiveness to BMP-7 treatment and no significant difference in total tubule perimeter was observed between untreated $\mathrm{HECV}^{\mathrm{RGMb} \mathrm{KO}}$ and $40 \mathrm{ng} / \mathrm{ml}$ BMP-7 treated HECV ${ }^{\text {RGMb KO }}$ cells ( $\left.\mathrm{p}=0.986\right)$. 
a

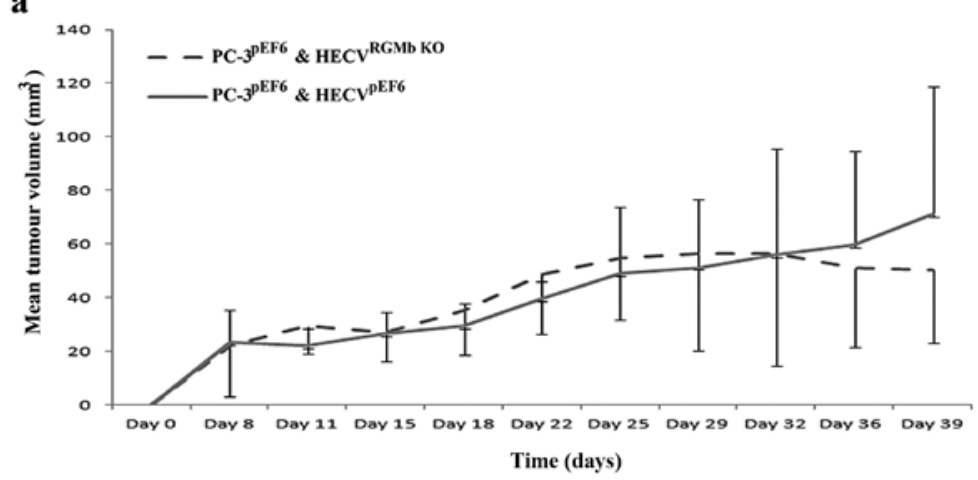

b

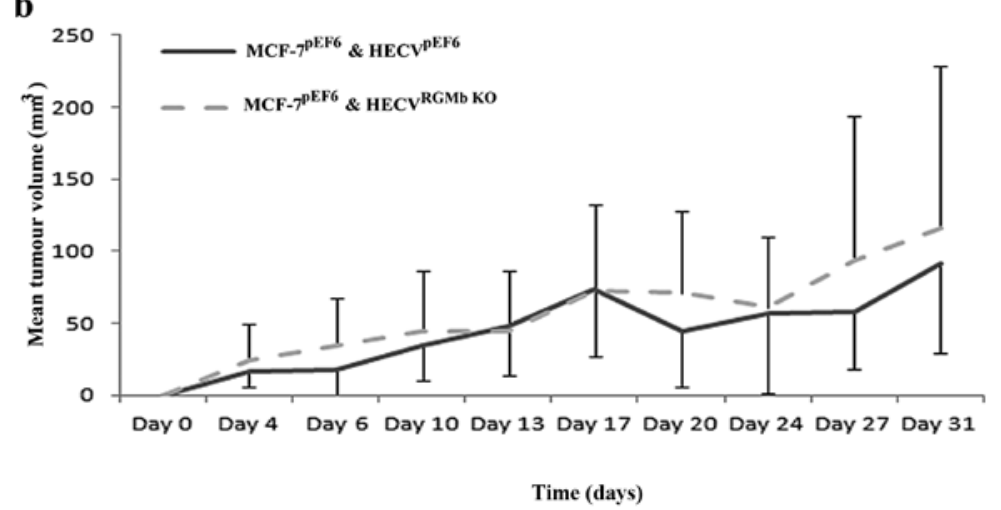

Figure 4. In vivo impact of RGMb knockdown. A co-inoculation angiogenesis assay was used to compare the ability of $\mathrm{HECV}^{\mathrm{pEF} 6}$ control cells and $\mathrm{HECV}^{\mathrm{RGMb} \text { KO }}$ cells to contribute to tumour development, in CD-1 athymic nude mice, of PC-3 prostate cancer (a) and MCF-7 breast cancer (b) cells in vivo. No significant differences were seen in the development of PC-3 or MCF-7 tumour development between those co-inoculated with HECV ${ }^{\mathrm{pEF} 6}$ and those inoculated with $\mathrm{HECV}^{\mathrm{RGMb}}$ KO . Error bars represent SEM.
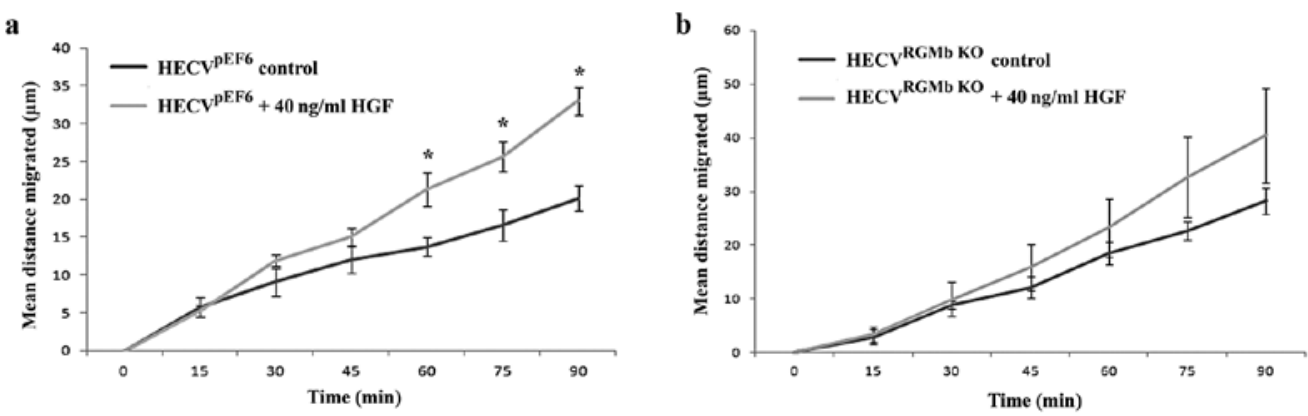

c

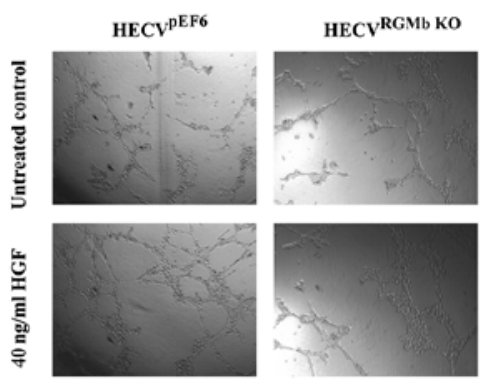

d

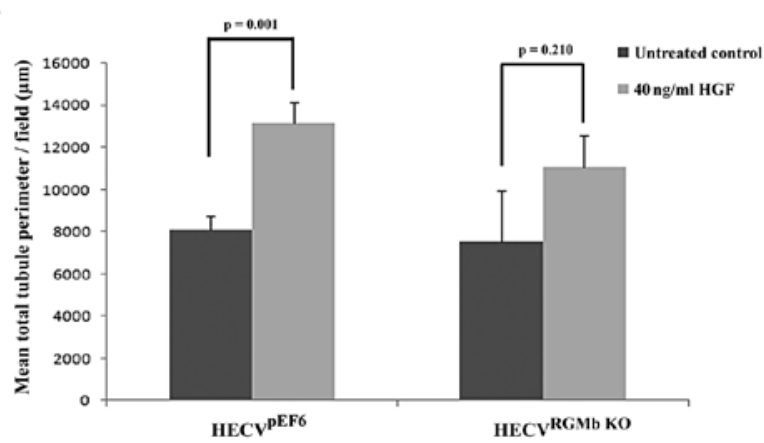

Figure 5. RGMb knockdown can suppress HGF mediated pro-angiogenic traits in HECV endothelial cells. (a) HGF treatment significantly enhanced HECV ${ }^{\mathrm{pEF}}$ cell migration compared to untreated $\mathrm{HECV}^{\mathrm{pEF} 6}$ cells. In contrast to this treatment of $\mathrm{HECV}^{\mathrm{RGMb} \text { KO }}$ cells with HGF, whilst bringing about a general increase in migration rates, was not found to have any significant impact in comparison to untreated $\mathrm{HECV}^{\mathrm{RGMb}}{ }^{\mathrm{KO}}$ cells (b). Similarly, treatment of $\mathrm{HECV}^{\mathrm{pEF} 6}$ cells with HGF could significantly enhance tubule formation in an in vitro Matrigel tubule formation assay in comparison to untreated HECV ${ }^{\mathrm{EFF} 6}$ control cells but only brought about a reduced, non-significant response in $\mathrm{HECV}^{\mathrm{RGMb} \mathrm{KO}}$ cells compared to untreated $\mathrm{HECV}^{\mathrm{RGM} \text { KO }}$ cells (c and d). Representative images are shown in (c). Error bars represent SEM; ${ }^{*} \mathrm{p}<0.05$. 
a
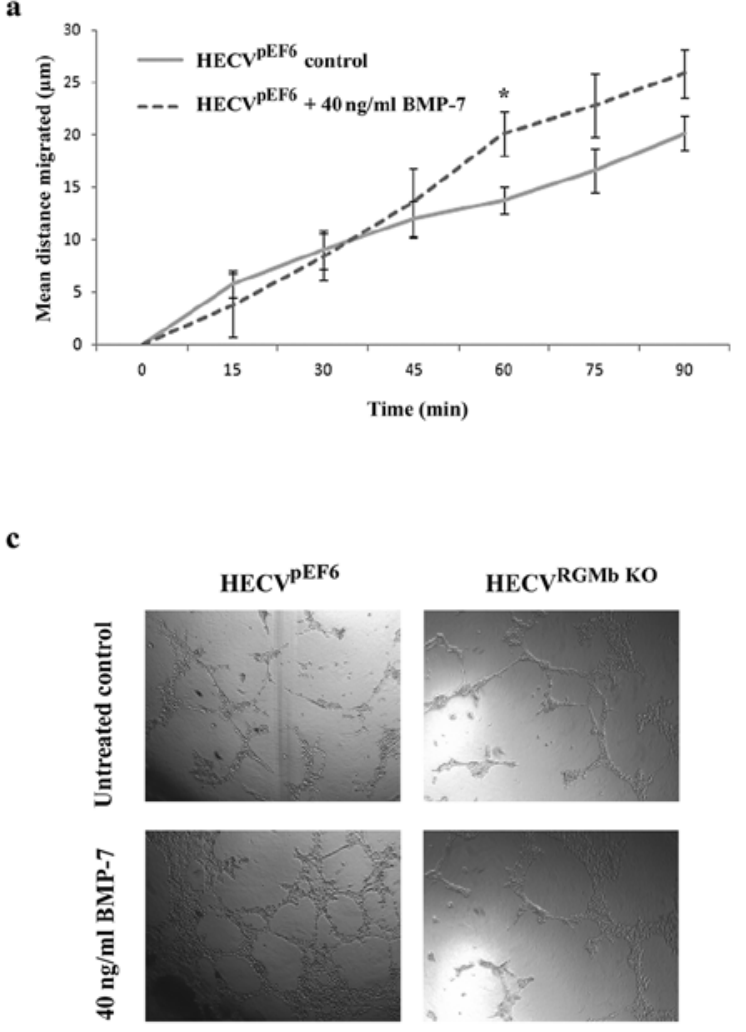

b

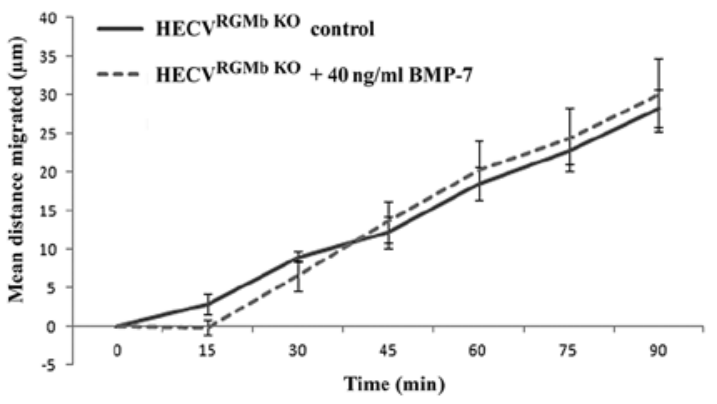

d

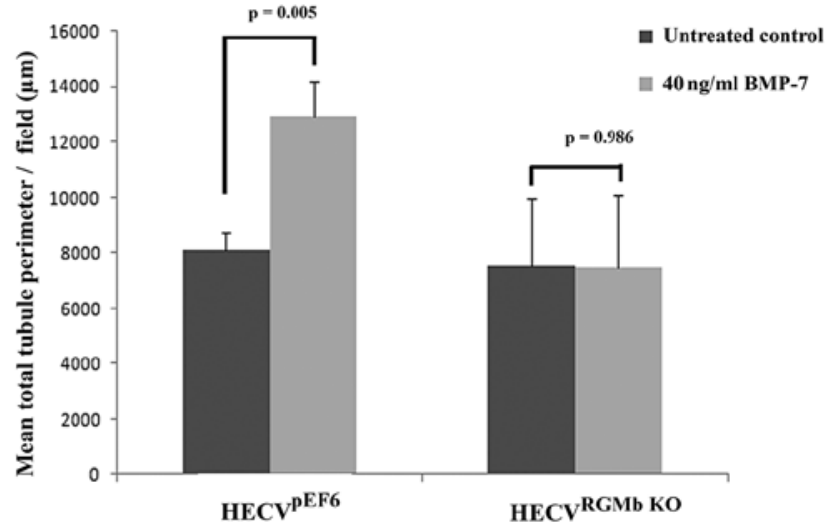

Figure 6. RGMb knockdown inhibits BMP-7-mediated pro-angiogenic response in HECV endothelial cells. (a) BMP-7 treatment partially enhanced the cell migration rates of $\mathrm{HECV}^{\mathrm{pEF} 6}$ control cells, resulting in significant or close to significant differences compared to untreated HECV ${ }^{\mathrm{pEF}}$ cells following 60-min incubation onwards. In contrast, virtually no deviations were observed between untreated HECV ${ }^{\mathrm{RGMb}} \mathrm{KO}$ cells and those treated with BMP-7 (b). Similar trends were observed in the Matrigel tubule formation assays where treatment with BMP-7 could enhance tubule formation levels of HECV cells, in comparison to untreated HECV ${ }^{\mathrm{pEF} 6}$ cells, but again demonstrated virtually no effects on HECV ${ }^{\mathrm{RGMb}} \mathrm{KO}$ cells treated with BMP-7 compared to those that remained untreated (c and d). Representative images are shown in (c). Error bars represent $\mathrm{SEM} ;{ }^{*} \mathrm{p}<0.05$.

\section{Discussion}

In the present study we used a microarray approach to identify gene expression that could be regulated by HGF and identified $\mathrm{RGMb}$ as a gene whose expression was enhanced $\sim 2.5$-fold following 4-h treatment with $40 \mathrm{ng} / \mathrm{ml}$ HGF. This data implicates RGMb as a HGF regulated gene in HECV human endothelial cells. Subsequently, we examined the potential of RGMb to impact on angiogenesis related factors in vitro and in vivo. RGMb expression was successfully targeted, using a ribozyme transgene system, in HECV cells resulting in a strong reduction in RGMb transcript and protein levels. Suppression of RGMb alone, however, did not appear to have substantial effects on HECV cells and only appeared to significantly influence cell migratory rates in the later stages of a scratch wounding migration assay. In vitro growth and tubule formation revealed no significant differences in RGMb suppressed compared to control HECV cells, though growth rates were elevated somewhat in the $\mathrm{HECV}^{\mathrm{RGMb} \text { KO }}$ cell line. Similar trends were observed in an in vivo model involving the co-inoculation of HECV endothelial cells and breast or prostate tumour cells suggesting that, solely, RGMb may contribute little to the angiogenic process. The results observed here regarding the role of RGMb in the basic function of endothe- lial cells are somewhat in line with previous work from our laboratories focusing on breast and prostate cancer. In both of these studies, knockout of RGMb resulted in enhanced migratory rates of prostate (PC-3) and breast (MDA-MB-231, MCF-7) cancer cell lines and enhanced cell growth rates in PC-3 and MDA-MB-231 cells, though not MCF-7 cells $(21,22)$. Together with our results the data suggest RGMb is involved in the regulation of cell migration and growth of certain cancer and to some extent endothelial cells.

Despite RGMb having minimal effect independently on these angiogenic traits, a role for RGMb was discovered in facilitating pro-angiogenic effects brought about by HGF. HECV cells displaying reduced RGMb expression were less able to respond in a pro-angiogenic manner to HGF treatment, and angiogenic traits such as cell migration and tubule formation capacity were not significantly enhanced by HGF treatment in $\mathrm{HECV}^{\mathrm{RGMb}} \mathrm{KO}$ cells as they were in HGF treated $\mathrm{HECV}^{\mathrm{p} E F 6}$ cells. Taken together with the establishment of HGFs capacity to regulate RGMb gene expression, our data provides evidence that RGMb may act as a potential mechanism to bring about HGFs pro-angiogenic effects.

In addition to examining the potential of RGMb to contribute to progressing the pro-angiogenic effects of HGF, and in light of the established roles of members of the RGM 
family as BMP co-receptors (14-17) we also aimed to explore the potential of RGMb to facilitate the pro-angiogenic effects of BMP-7. The BMPs are members of the transforming growth factor $\beta$ (TGF- $\beta$ ) family, exerting their signals through type I and II transmembrane serine/theronine kinase receptors to influence a plethora of biological processes including angiogenesis. BMP-2, $-4,-6$ and -7 have been implicated in both the activation phase, where they can enhance endothelial cell proliferation and migration, and also the maturation phase, where they can influence vascular smooth muscle cells (reviewed in refs. 30 and 31). Interestingly, suppression of RGMb in HECV cells, whilst reducing the pro-angiogenic effects of HGF, was also found to substantially inhibit BMP-7 promoted cell migration and tubule formation. The pro-migratory impact of BMP-7 itself was not as great as that of HGF, a well established motogen (3), with BMP-7 enhancement of migration rates not showing as significant increases in migration rates of control HECV cells as HGF. However, suppression of RGMb in HECV cells could substantially remove any pro-migratory effect of BMP-7 with migration rates showing similar levels to that of untreated $\mathrm{HECV}^{\mathrm{RGMb} \text { KO }}$ controls. In keeping with this trend a similar pattern was seen on tubule formation capacity. In control HECV $\mathrm{V}^{\mathrm{pEF}}$ cells, treatment with $40 \mathrm{ng} / \mathrm{ml}$ BMP-7 brought about a significant increase in tubule formation levels. Suppression of RGMb again inhibited this pro-tubule formation effect with mean tubule formation levels comparable to untreated controls and no significant difference being observed between untreated and treated HECV ${ }^{\mathrm{RGMb} \mathrm{KO}}$ cells. The data appear to be in line with the current literature, identifying RGMs as BMP co-receptors (14-17) and this study demonstrates the importance of this in contributing to the angiogenic process.

It is noteworthy that HGF has previously been demonstrated to enhance the expression of BMP-7 and the expression of the BMPR-IB and BMPR-II BMP receptors in prostate cancer cells $(32,33)$. A similar trend has also been observed briefly in the HECV endothelial cells used in this study, where treatment with HGF was found, using qPCR, to enhance BMP-7 levels in a time course experiment (data not shown). It may be worth considering that crosstalk between these two pathways may present a partial means to explain the results obtained in our study. A weaker response was apparent, particularly in the tubule formation experiments, in RGMb knockdown cells to BMP-7 rather than HGF. It is possible that HGF may partially act to enhance BMP signalling, possibly through enhancement of BMP-7 and/or BMP receptor levels in these cells, which in turn could be interrupted through RGMb suppression. This however, requires further scientific examination before conclusions can be drawn.

Other studies conducted in our laboratories have implicated members of the RGM family in breast and prostate cancer. Knockdown of RGMb was found to enhance PC-3 prostate cancer cell growth, cell-matrix adhesion and migration. Additionally, knockdown of RGMb enhanced levels of activated Smad 3 and ID1 expression, a trend that was increased through treatment with BMP-7, but generally reduced activated levels of Smad 1 (21). Similar to the prostate cancer study, knockdown of RGMb in MDA-MB-231 breast cancer cells was also found to enhance cell growth, matrix adhesion and migration. Knockdown of RGMb was also found to facilitate survival from apoptosis under serum starvation. Alterations, following RGMb knockdown, were observed in the regulation of c-myc, caspase-3, SNAIL, TWIST, FAK and paxillin. This study also implicated RGMb knockdown to contribute to the Smad-dependent pathway, enhancing levels of activated Smad 1 and 3 in untreated cells, with greater responses seen following BMP-7 treatment and a switching to Smad 1 activation following inhibition of Smad 3, whereas phosphorylation of JNK, ILP and TAK was inhibited following RGMb knockdown suggesting suppression of the Smad-independent pathway (22).

In the present study, knockdown of RGMb in human endothelial cells enhanced migration and also appeared to suppress the pro-angiogenic response to HGF and BMP-7. Taken together, this suggests that, while loss of RGMb may enhance aggressive traits in prostate and breast cancer cells, suppression of RGMb may also act to suppress HGF and BMP-7 mediated tumour angiogenesis. Also, in contrast to the previous prostate and breast cancer studies, where treatment of RGMb knockdown cells with BMP-7 enhances activity of Smad 1 or 3, our present study implies that in HECV cells RGMb knockdown may act to suppress signalling resulting from BMP-7 treatment. RGMb has been identified as a BMP co-receptor, directly binding BMP-2, and -4 but not -7 or TGF- $\beta$ ligands and associating with the ALK2, 3 and 6, BMP type I and the ActRII and ActRIIB BMP type II receptors (14). Since its discovery a number of studies have shown this molecule to play a role in enhancing BMP signalling (12, 14, 18). However, RGMb has also demonstrated the ability to inhibit BMP signalling in $\mathrm{C} 2 \mathrm{C} 12$ myoblasts (34). These observations, taken with the data presented here and the previous prostate and breast cancer studies $(21,22)$ suggest a complex relationship between RGMb and BMP signalling. Further scientific study is required to establish fully the downstream effectors of BMP-7 and HGF treatment in endothelial cells. The data presented in this study have, for the first time, raised the implication that RGMb may play some complex role(s) in the process of tumour angiogenesis mediated by HGF and BMP-7 and further links RGMb to tumour progression.

\section{Acknowledgements}

The authors wish to thank Cancer Research Wales and the Henry Fong Family Foundation for supporting this study.

\section{References}

1. Folkman J: Angiogenesis: an organizing principle for drug discovery? Nat Rev Drug Discov 6: 273-286, 2007.

2. Potente M, Gerhardt $\mathrm{H}$ and Carmeliet P: Basic and therapeutic aspects of angiogenesis. Cell 146: 873-887, 2011.

3. Jiang WG, Martin TA, Parr C, Davies G, Matsumoto K and Nakamura T: Hepatocyte growth factor, its receptor, and their potential value in cancer therapies. Crit Rev Oncol Hematol 53: 35-69, 2005.

4. Wojta J, Kaun C, Breuss JM, et al: Hepatocyte growth factor increases expression of vascular endothelial growth factor and plasminogen activator inhibitor-1 in human keratinocytes and the vascular endothelial growth factor receptor flk-1 in human endothelial cells. Lab Invest 79: 427-438, 1999.

5. Davies G, Mason MD, Martin TA, et al: The HGF/SF antagonist NK4 reverses fibroblast- and HGF-induced prostate tumor growth and angiogenesis in vivo. Int J Cancer 106: 348-354, 2003. 
6. Martin TA, Parr C, Davies G, et al: Growth and angiogenesis of human breast cancer in a nude mouse tumour model is reduced by NK4, a HGF/SF antagonist. Carcinogenesis 24: 1317-1323, 2003.

7. Monnier PP, Sierra A, Macchi P, et al: RGM is a repulsive guidance molecule for retinal axons. Nature 419: 392-395, 2002

8. Oldekamp J, Kramer N, Alvarez-Bolado G and Skutella T: Expression pattern of the repulsive guidance molecules RGM $\mathrm{A}, \mathrm{B}$ and $\mathrm{C}$ during mouse development. Gene Expr Patterns 4 283-288, 2004

9. Schmidtmer J and Engelkamp D: Isolation and expression pattern of three mouse homologues of chick Rgm. Gene Expr Patterns 4: 105-110, 2004.

10. Niederkofler V, Salie R, Sigrist M and Arber S: Repulsive guidance molecule (RGM) gene function is required for neural tube closure but not retinal topography in the mouse visual system. J Neurosci 24: 808-818, 2004.

11. Samad TA, Srinivasan A, Karchewski LA, et al: DRAGON a member of the repulsive guidance molecule-related family of neuronal- and muscle-expressed membrane proteins is regulated by DRG11 and has neuronal adhesive properties. J Neurosci 24 2027-2036, 2004.

12. Ma CH, Brenner GJ, Omura T, et al: The BMP coreceptor RGMb promotes while the endogenous BMP antagonist noggin reduces neurite outgrowth and peripheral nerve regeneration by modulating BMP signaling. J Neurosci 31: 18391-18400, 2011.

13. Liu X, Hashimoto M, Horii H, Yamaguchi A, Naito K and Yamashita T: Repulsive guidance molecule b inhibits neurite growth and is increased after spinal cord injury. Biochem Biophys Res Commun 382: 795-800, 2009.

14. Samad TA, Rebbapragada A, Bell E, et al: DRAGON, a bone morphogenetic protein co-receptor. J Biol Chem 280 14122-14129, 2005.

15. Babitt JL, Zhang Y, Samad TA, et al: Repulsive guidance molecule (RGMa), a DRAGON homologue, is a bone morphogenetic protein co-receptor. J Biol Chem 280: 29820-29827, 2005.

16. Babitt JL, Huang FW, Wrighting DM, et al: Bone morphogenetic protein signaling by hemojuvelin regulates hepcidin expression. Nat Genet 38: 531-539, 2006.

17. Halbrooks PJ, Ding R, Wozney JM and Bain G: Role of RGM coreceptors in bone morphogenetic protein signaling. J Mol Signal 2: 4, 2007.

18. Xia Y, Sidis Y, Mukherjee A, et al: Localization and action of Dragon (repulsive guidance molecule b), a novel bone morphogenetic protein coreceptor, throughout the reproductive axis. Endocrinology 146: 3614-3621, 2005.

19. Xia Y, Cortez-Retamozo V, Niederkofler V, et al: Dragon (repulsive guidance molecule b) inhibits IL-6 expression in macrophages. J Immunol 186: 1369-1376, 2011.

20. Li J, Ye L, Mansel RE and Jiang WG: Potential prognostic value of repulsive guidance molecules in breast cancer. Anticancer Res 31: 1703-1711, 2011.
21. Li J, Ye L, Kynaston HG and Jiang WG: Repulsive guidance molecules, novel bone morphogenetic protein co-receptors, are key regulators of the growth and aggressiveness of prostate cancer cells. Int J Oncol 40: 544-550, 2012.

22. Li J, Ye L, Sanders AJ and Jiang WG: Repulsive guidance molecule B (RGMB) plays negative roles in breast cancer by coordinating BMP signaling. J Cell Biochem 113: 2523-2531, 2012.

23. Sanders AJ, Parr C, Mason MD and Jiang WG: Suppression of hepatocyte growth factor activator inhibitor-1 leads to a more aggressive phenotype of prostate cancer cells in vitro. Int J Mol Med 20: 613-619, 2007.

24. Yuan Z, Sanders AJ, Ye L, Wang Y and Jiang WG: Knockdown of human antigen $\mathrm{R}$ reduces the growth and invasion of breast cancer cells in vitro and affects expression of cyclin D1 and MMP-9. Oncol Rep 26: 237-245, 2011.

25. Zuker M: Mfold web server for nucleic acid folding and hybridization prediction. Nucleic Acids Res 31: 3406-3415, 2003.

26. Parr C, Sanders AJ, Davies G, et al: Matriptase-2 inhibits breast tumor growth and invasion and correlates with favorable prognosis for breast cancer patients. Clin Cancer Res 13: 3568-3576, 2007.

27. Parr C, Watkins G, Mansel RE and Jiang WG: The hepatocyte growth factor regulatory factors in human breast cancer. Clin Cancer Res 10: 202-211, 2004.

28. Jiang WG, Hiscox SE, Parr C, et al: Antagonistic effect of NK4, a novel hepatocyte growth factor variant, on in vitro angiogenesis of human vascular endothelial cells. Clin Cancer Res 5: 3695-3703, 1999

29. Sanders AJ, Ye L, Mason MD and Jiang WG: The impact of EPLINalpha (Epithelial protein lost in neoplasm) on endothelial cells, angiogenesis and tumorigenesis. Angiogenesis 13: 317-326, 2010.

30. David L, Feige JJ and Bailly S: Emerging role of bone morphogenetic proteins in angiogenesis. Cytokine Growth Factor Rev 20: 203-212, 2009.

31. Cai J, Pardali E, Sanchez-Duffhues G and ten Dijke P: BMP signaling in vascular diseases. FEBS Lett 586: 1993-2002, 2012.

32. Ye L, Lewis-Russell JM, Davies G, Sanders AJ, Kynaston H and Jiang WG: Hepatocyte growth factor up-regulates the expression of the bone morphogenetic protein (BMP) receptors, BMPR-IB and BMPR-II, in human prostate cancer cells. Int J Oncol 30: 521-529, 2007.

33. Ye L, Lewis-Russell JM, Sanders AJ, Kynaston H and Jiang WG: $\mathrm{HGF} / \mathrm{SF}$ up-regulates the expression of bone morphogenetic protein 7 in prostate cancer cells. Urol Oncol 26: 190-197, 2008.

34. Kanomata K, Kokabu S, Nojima J, Fukuda T and Katagiri T: DRAGON, a GPI-anchored membrane protein, inhibits BMP signaling in C2C12 myoblasts. Genes Cells 14: 695-702, 2009. 